\title{
Fauna de Coleoptera no Parque Estadual de Vila Velha, Ponta Grossa, Paraná, Brasil. Abundância e riqueza das famílias capturadas através de armadilhas de solo ${ }^{1}$
}

\author{
Renato C. Marinoni ${ }^{2,3}$ \& Norma G. Ganho ${ }^{2,4}$ \\ ${ }^{1}$ Contribuição número 1433 do Departamento de Zoologia, Universidade Federal do Paraná. \\ 2 Departamento de Zoologia, Universidade Federal do Paraná. Caixa Postal 19020, 81531-980 Curitiba, Paraná, Brasil. \\ ${ }^{3}$ Bolsista do CNPq. E-mail: rcmari@ufpr.br \\ ${ }^{4}$ Bolsista da CAPES. E-mail: jomario@uol.com.br
}

\begin{abstract}
Coleoptera fauna in the Parque Estadual de Vila Velha, Ponta Grossa, Paraná, Brazil. Abundance and family richness captured with pitfall traps. This paper is a part of the studies on the Coleoptera fauna from Vila Velha, Ponta Grossa, Parana gathered through malaise and pitfall traps in sites with different floristic conditions. The present study deal with the data of pitfall trap captures, installed close to the malaise traps. The data were obtained weekly (52 samples), from September 1999 to August 2000. This survey was carried out on five areas, three of them with different plant succession stages (initial, intermediate, and advanced). The other two sites were: in Araucaria angustifolia plantation area, with the understory invaded by native forest vegetation and in the edge area. In the last one, two pitfall traps were installed, one inside the forest and other in the field vegetation. The Coleoptera communities were analyzed according the abundance, family richness, and the floristic conditions of the sites. The total of specimens collected was 13,093 belonging to 35 families. The most abundant site was the one in initial stage of succession; the abundance was lowest in the edge area. The beetle faunal composition of the five sites, including all the families, were not significantly different. However, when listed only the seven dominant families, the results showed the outside edge fauna composition significantly different from the other sites. Among the most abundant families in the Vila Velha litter are Staphylinidae, Ptiliidae, Nitidulidae, Scarabaeidae, Scolytidae, Hydrophilidae and Endomychidae, eventually substituted by Latridiidae, Corylophidae, Curculionidae, Carabidae and Histeridae. Several comparisons were made with the data from malaise and pitfall traps data obtained in Vila Velha, and from other studies in several world regions. From these comparisons was possible to highlight: the beetle family richness is higher when captured by malaise than pitfall trap; the family taxonomic compositions in the litter from several regions of the world are more similar among themselves than the taxonomic compositions from malaise captures in the same region; a small number of beetle families (five to seven) is liable by approximately $60 \%$ of the total abundance in the malaise, and about $90 \%$ in the litter; the majority litter faunal surveys showed non-herbivorous families as dominants, while in the malaise trap the herbivorous families are dominants; there are evidences that certain taxa are substituted by others of the same trophic group by ecologic reasons when in the same region, and by zoogeographic reasons in different regions.
\end{abstract}

KEY WORDS. Araucaria forest, family composition, family constancy, leaf litter, vegetation succession, trophic group.

No trabalho de GANHO \& MARINONI (2003) foi apresentado um estudo inicial sobre a fauna de Coleoptera de Vila Velha, Ponta Grossa, produto de um levantamento realizado durante o período de setembro de 1999 a agosto de 2000. Fez-se, então, uma análise da abundância e riqueza das famílias capturadas por armadilha malaise, em cinco áreas com diferentes características vegetais, produto de alterações ambientais antrópicas. No presente trabalho, apresenta-se um estudo envolvendo a fauna de coleópteros capturados através de armadilha de solo, nos mesmos ambientes e período.
Vários trabalhos têm sido produzidos buscando estabelecer relações entre a fauna de Coleoptera de solo e o meio ambiente. Com este objetivo e utilizando diferentes métodos de captura, encontram-se na literatura, por exemplo, os trabalhos de Schubart \& Beck (1968), Moeed \& Meads (1985), Rodrigues (1992), Carlton \& Robison (1998), Didham et al. (1998a, b) e Chung et al. (2000).

Nos diferentes estudos sobre a fauna de Coleoptera de solo, os parâmetros utilizados para as análises têm variado. SCHUbart \& Beck (1968) fizeram uma comparação da fauna de 
solo de diferentes biótopos empregando a abundância de indivíduos por família, como o fez Rodrigues (1992). Davies et al. (1997), Carlton \& Robison (1998), Didham et al. (1998a, b), Chung et al. (2000) e BARBOSA et al. (2002) utilizaram o número de espécies por família como elemento de comparação nas análises, além do número de indivíduos.

Tem sido observado que, no mesmo estrato florestal de várias regiões do mundo, há uma constância na composição taxonômica de famílias de Coleoptera, opondo-se à constatação de que em um mesmo ambiente, diferentes estratos apresentam composições taxonômicas diferentes (DAVIES et al. 1997, GANHO \& MARINONI 2003). Estas características têm sido descritas para o dossel e para o estrato que corresponde ao espaço entre o solo e cerca de 1,20 m (parte do sub-bosque mais vegetação rasteira) e no qual os Coleoptera estão sujeitos à captura por armadilha malaise.

Os estudos da fauna de Coleoptera envolvendo apenas as famílias, ou espécies, mais abundantes é uma forma de minimizar as dificuldades de identificação dos táxons mais raros e acelerar as análises. Desta forma, é importante que se procure levantar o maior número de informações sobre as diferentes relações entre os besouros e o meio ambiente, neste nível de organização taxonômica, para consolidar o grupo como possível indicador de condições ambientais em áreas florestadas.

Neste trabalho foi feito um estudo sobre a abundância e riqueza da fauna de Coleoptera, capturada através de armadilha de solo, a partir do número de indivíduos e famílias, em áreas com diferentes graus de antropização e de sucessão vegetal, no Parque Estadual de Vila Velha, em Ponta Grossa, Estado do Paraná, procurando evidenciar as relações inter-áreas da fauna de Coleoptera decorrentes das diferenças florísticas; reconhecer a constância da presença de famílias capturadas por armadilha de solo, em diferentes locais e/ou regiões, entre aquelas com os mais altos valores de abundância; acrescer informações sobre a viabilidade do uso das famílias mais abundantes para análises de estrutura de comunidade de Coleoptera e de suas relações com o meio.

\section{MATERIAL E MÉTODOS}

O inventário foi realizado no Parque Estadual de Vila Velha, localizado no município de Ponta Grossa, Estado do Paraná, junto à Rodovia do Café, BR 376, Km 83, a 880 m de altitude, utilizando armadilha de solo, de setembro de 1999 a agosto de 2000 .

\section{Características do local e dos pontos de coleta}

O detalhamento destas características e considerações sobre as condições da flora encontram-se em GANHO \& MARINONI (2003).

Área de borda $\left(25^{\circ} 13^{\prime} 5,0^{\prime \prime} \mathrm{S}, 50^{\circ} 2^{\prime} 26,9^{\prime \prime} \mathrm{W}\right)$. Caracterizava-se como de transição entre a vegetação arbórea em estágio intermediário de sucessão e área de campo. Esta última mantida por roçadura (pelo menos semestralmente), exceto por uma faixa de $10 \mathrm{~m}$ de raio em torno da armadilha, que permaneceu intocada durante o período. Uma armadilha de solo estava instalada na área de campo (denominada borda externa), a cerca de $5 \mathrm{~m}$ da borda; e outra na área florestada (denominada borda interna), a cerca de $10 \mathrm{~m}$ da borda.
Área de araucária $\left(25^{\circ} 13^{\prime} 5,9^{\prime \prime} \mathrm{S}, 50^{\circ} 2^{\prime} 31,2^{\prime \prime} \mathrm{W}\right)$. Armadilha situada a $240 \mathrm{~m}$ da área de borda, aproximadamente. Trata-se de um povoamento florestal de Araucaria angustifolia (Bertol.) Kuntze (pinheiro-do-paraná), que teve o manejo abandonado por volta de 1981 .

Área fase $1\left(25^{\circ} 13^{\prime} 13,3^{\prime \prime} \mathrm{S}, 50^{\circ} 2^{\prime} 14,1^{\prime \prime} \mathrm{W}\right)$. Armadilha situada a $335 \mathrm{~m}$ da área de borda, aproximadamente. Área com cerca de 15 ha, anteriormente utilizada para culturas agrícolas sazonais, como milho e feijão. Em processo de regeneração natural desde 1984. Encontra-se numa fase inicial a intermediária de sucessão vegetal.

Área fase $2\left(25^{\circ} 13^{\prime} 2,9^{\prime \prime} \mathrm{S}, 50^{\circ} 2^{\prime} 14,1^{\prime \prime} \mathrm{W}\right)$. Armadilha situada a $400 \mathrm{~m}$ da área de borda, aproximadamente. Floresta primária alterada pela retirada de várias essências vegetais, como pinheiro-do-paraná, imbuia, canelas diversas e algumas mirtáceas. A sucessão vegetal, dependendo do ponto observado, varia de intermediária a avançada.

Área fase $3\left(25^{\circ} 13^{\prime} 27,6^{\prime \prime} \mathrm{S}, 50^{\circ} 1^{\prime} 52,7^{\prime \prime} \mathrm{W}\right)$. Armadilha situada a $1200 \mathrm{~m}$ das armadilhas da área de borda e a $90 \mathrm{~m}$ da borda da floresta, aproximadamente. Floresta primária alterada por cortes seletivos. É a mais bem preservada dentre todas, com a estrutura definida e homogênea, com a flora muito semelhante à da área fase 2 .

\section{Método de coleta}

Foram utilizadas armadilhas de solo (Almeida et al. 1998) com diâmetro de $15 \mathrm{~cm}$ de diâmetro, sem atrativos. Este tipo de armadilha foi escolhido por coletar permanentemente insetos que vivem e caminham no solo, principalmente entre o folhiço. O material era retirado às segundas-feiras pela manhã do frasco coletor, contendo formol a $4 \%$, e transferido para recipientes devidamente identificados.

\section{Material e identificação}

Os exemplares, depois de montados em alfinetes entomológicos, e devidamente etiquetados, foram separados em morfoespécies e identificados ao nível de família, conforme descrito em GanHo \& Marinoni (2003). As referências aos hábitos alimentares das famílias foram extraídas de MARINONI et al. (2001). O material coletado foi depositado na Coleção de Entomologia Pe. Jesus Santiago Moure.

\section{Análises estatísticas e de agrupamento}

As análises estatísticas foram feitas utilizando o programa Statistica 6 StatSoft, Inc. (2001). Para as análises de agrupamento foi utilizado o programa NTSYS-pc (Numerical Taxonomy and Multivariate Analysis System) 2.02, desenvolvido por Rohlf (1998). Os diferentes métodos empregados são indicados no texto.

Ao longo do texto, as várias comparações e discussões com os dados de captura através de armadilha malaise, obtidos nas mesmas áreas e ocasião, em Vila Velha, foram apresentados em GanHo \& Marinoni (2003).

\section{RESULTADOS E DISCUSSÃO}

\section{Abundância}

Nas seis armadilhas de solo foram capturados 13.093 exemplares (Tab. I). O maior número indivíduos (3.205) foi capturado na área fase 1 e o menor na área de borda (856). 
Tabela I. Famílias de Coleoptera capturadas com armadilha de solo em cinco áreas do Parque Estadual de Vila Velha, Ponta Grossa, Paraná, durante o período de setembro de 1999 a agosto de 2000, com número de indivíduos.

\begin{tabular}{|c|c|c|c|c|c|c|c|}
\hline \multirow{2}{*}{ Família } & \multicolumn{2}{|c|}{ Borda } & \multirow{2}{*}{ Araucária } & \multirow{2}{*}{ Fase 1} & \multirow{2}{*}{ Fase 2} & \multirow{2}{*}{ Fase 3} & \multirow{2}{*}{ Total } \\
\hline & Externa (BE) & Interna (BI) & & & & & \\
\hline Anobiidae & 0 & 0 & 1 & 0 & 0 & 0 & 1 \\
\hline Carabidae & 14 & 26 & 5 & 21 & 5 & 9 & 80 \\
\hline Cerambycidae & 0 & 5 & 5 & 11 & 2 & 4 & 27 \\
\hline Chrysomelidae & 7 & 1 & 1 & 5 & 3 & 5 & 22 \\
\hline Cleridae & 0 & 0 & 0 & 1 & 0 & 1 & 2 \\
\hline Coccinellidae & 1 & 0 & 0 & 0 & 0 & 0 & 1 \\
\hline Corylophidae & 42 & 8 & 2 & 18 & 8 & 1 & 79 \\
\hline Cryptophagidae & 0 & 0 & 0 & 1 & 0 & 1 & 2 \\
\hline Cucujidae & 4 & 10 & 8 & 3 & 4 & 2 & 31 \\
\hline Curculionidae & 34 & 19 & 1 & 11 & 10 & 1 & 76 \\
\hline Dermestidae & 0 & 1 & 0 & 0 & 0 & 0 & 1 \\
\hline Elateridae & 5 & 13 & 3 & 2 & 5 & 1 & 29 \\
\hline Endomychidae & 5 & 17 & 41 & 33 & 29 & 13 & 138 \\
\hline Histeridae & 5 & 6 & 23 & 17 & 40 & 8 & 99 \\
\hline Hydrophilidae & 21 & 39 & 13 & 33 & 27 & 28 & 161 \\
\hline Lampyridae & 0 & 1 & 0 & 1 & 0 & 0 & 2 \\
\hline Lathridiidae & 101 & 10 & 2 & 6 & 3 & 3 & 125 \\
\hline Leiodidae & 7 & 2 & 1 & 4 & 16 & 1 & 31 \\
\hline Melandryidae & 0 & 0 & 0 & 0 & 0 & 1 & 1 \\
\hline Monotomidae & 15 & 0 & 0 & 1 & 1 & 0 & 17 \\
\hline Mordelidae & 3 & 1 & 0 & 0 & 0 & 0 & 4 \\
\hline Mycetophagidae & 1 & 0 & 0 & 0 & 0 & 1 & 2 \\
\hline Nemonychidae & 0 & 1 & 0 & 0 & 0 & 0 & 1 \\
\hline Nitidulidae & 242 & 156 & 138 & 84 & 263 & 183 & 1066 \\
\hline Passalidae & 0 & 0 & 0 & 1 & 0 & 0 & 1 \\
\hline Phalacridae & 0 & 0 & 1 & 0 & 0 & 0 & 1 \\
\hline Platypodidae & 0 & 3 & 0 & 2 & 0 & 5 & 10 \\
\hline Ptiliidae & 21 & 157 & 418 & 749 & 459 & 628 & 2432 \\
\hline Ptilodactylidae & 6 & 3 & 1 & 1 & 3 & 0 & 14 \\
\hline Scarabaeidae & 43 & 70 & 94 & 106 & 92 & 62 & 467 \\
\hline Scolytidae & 27 & 90 & 107 & 49 & 128 & 91 & 492 \\
\hline Scydemaenidae & 14 & 3 & 4 & 13 & 4 & 8 & 46 \\
\hline Silvanidae & 19 & 0 & 3 & 1 & 1 & 1 & 25 \\
\hline Staphylinidae & 215 & 972 & 1498 & 2019 & 1063 & 1792 & 7559 \\
\hline Tenebrionidae & 4 & 4 & 0 & 12 & 21 & 7 & 48 \\
\hline Total de indivíduos & 856 & 1618 & 2370 & 3205 & 2187 & 2857 & 13093 \\
\hline Total de famílias & 24 & 25 & 22 & 27 & 22 & 25 & 35 \\
\hline Total de famílias BE e BI & & 29 & & & & & \\
\hline
\end{tabular}


A abundância foi maior nas áreas florestadas que na área de borda, tanto na coleta por armadilha malaise quanto por armadilha de solo. OzanNe et al. (1997) também verificaram haver, em área de borda, uma menor abundância de artrópodos no dossel de coníferas. Ao contrário, Didham et al. (1998a, b), que observaram vários aspectos da dinâmica dos Coleoptera em relação aos efeitos de borda e fragmentação em floresta tropical, concluíram que a densidade dos coleópteros aumentou em direção à borda.

Dentre as áreas em sucessão natural, a menor abundância foi observada na fase 2 que, no entanto, foi a mais abundante nas coletas por malaise. Esta menor abundância foi produto do baixo número de Staphylinidae, quase a metade da abundância da família observada na área fase 1 . O elemento mais destacado do ambiente, a ser considerado como uma possível causa na notável queda de abundância, era o maior sombreamento do solo na área, devido ao dossel se apresentar como o mais fechado dentre todas elas, refletindo-se na presença de poucas gramíneas e plantas arbustivas. Assim, pelo menos quando se trata de Staphylinidae, a diminuição da abundância não corresponderia ao que Hambler \& SPEIGHT (1995, segundo Chung et al. 2000) postularam para os artrópodos, ao afirmarem que baixo nível de luminosidade ao nível do solo está correlacionado com uma alta biomassa dessa comunidade.

\section{Riqueza das famílias}

Segundo Costa (2000), 104 famílias de Coleoptera, das 166 listadas por LaWrence \& Newton (1995), estão presentes no Brasil. No levantamento feito em Vila Velha, Ponta Grossa, foram capturadas 67 famílias, quando são arroladas as capturas por armadilha de solo e por armadilha Malaise, ou seja, $40 \%$ das famílias conhecidas mundialmente e $64 \%$ das conhecidas no Brasil.

O total de famílias capturadas no solo, em todas as áreas, foi de 35. Na área fase 1 foi observada a maior riqueza com 27 famílias, seguida da área fase 3 e da área de borda interna com 25. Considerando-se as duas áreas de borda juntas (externa e interna) foram coletadas 29 famílias. As áreas de araucária e fase 2 , foram as que apresentaram a menor riqueza, com 22 famílias.

Estes números são semelhantes aos observados nas coletas realizadas no extrato do solo, em diferentes regiões do mundo. No Brasil (Amazônia), Malásia e Nova Zelândia, as coletas de coleópteros do solo, sejam através de armadilhas ou extração do folhiço, resultaram no reconhecimento de 30 a 42 famílias por levantamento, com os valores por ponto de coleta variando entre 20 e 30 famílias (Schubart \& Beck 1968, Moeed \& Meads 1985, Rodrigues 1992, Didham et al. 1998b, Chung et al. 2000, Barbosa et al. 2002). O número de famílias coletadas no solo de diferentes áreas em diferentes regiões é quase sempre inferior ao número de famílias produto da captura por armadilha malaise, que foi de 41 a 64 famílias, conforme dados levantados por GANHO \& MARINONI (2003).

\section{Estrutura de comunidades}

As estruturas das comunidades de Coleoptera dos seis diferentes pontos de coleta em Vila Velha, Ponta Grossa, baseadas nos valores de abundância das famílias (Tab. I) não foram significativamente diferentes (Coeficiente de correlação linear, p < 0,05) (Tab. II).

Mas, como esperado, as estruturas mais semelhantes fo- ram as das áreas florestadas, conforme representado pela figura 1 (análise de agrupamento; coeficiente de correlação linear; UPGMA; coeficiente de correlação cofenética $=0,987$ ).

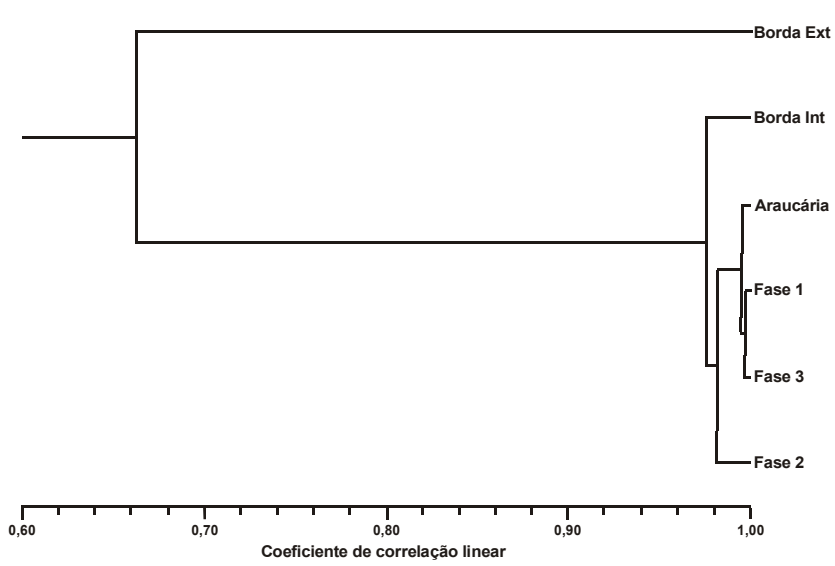

Figura 1. Análise de agrupamento. Relação entre os seis pontos de coleta, por armadilha de solo, no Parque Estadual Vila Velha, Ponta Grossa, Paraná, durante o período de setembro de 1999 a agosto de 2000, com base nos dados de abundância das famílias de Coleoptera.

Tabela II. Coeficiente de correlação linear entre dados de abundância de todas as famílias de Coleoptera coletadas por armadilha de solo, nos seis pontos de coleta no Parque Estadual Vila Velha, Ponta Grossa, Paraná, durante o período de setembro de 1999 a agosto de 2000. $\left(^{*}\right)$ Correlação significativa $(p<0,05)$ entre todos os pontos.

\begin{tabular}{|c|c|c|c|c|c|c|}
\hline \multirow{2}{*}{ Áreas } & \multicolumn{2}{|c|}{ Borda } & \multirow{2}{*}{ Araucária } & \multirow{2}{*}{ Fase 1} & \multirow{2}{*}{ Fase 2} & \multirow{2}{*}{ Fase 3} \\
\hline & Externa & Interna & & & & \\
\hline Borda Externa & - & & & & & \\
\hline Borda Interna & $0,7115^{*}$ & - & & & & \\
\hline Araucária & $0,6501^{*}$ & $0,9896^{*}$ & - & & & \\
\hline Fase 1 & $0,6017^{*}$ & $0,9696^{*}$ & $0,9937^{*}$ & - & & \\
\hline Fase 2 & $0,7093^{*}$ & $0,9656^{*}$ & $0,9808^{*}$ & $0,9774^{*}$ & - & \\
\hline Fase 3 & $0,6435^{*}$ & $0,9792^{*}$ & $0,9968^{*}$ & $0,9975^{*}$ & $0,9866^{*}$ & - \\
\hline
\end{tabular}

A fase 2 aparece como a mais diferenciada dentre as áreas florestadas, resultado da relativamente fraca abundância da família Staphylinidae, já destacado acima. A área de borda externa, apesar da estrutura de comunidade de Coleoptera não ser significativamente diferente das demais áreas, foi a que se apresentou como a mais distinta de todas as outras, quando a análise considerou todas as famílias (das abundantes às raras).

Esta diferenciação é coerente com as condições bióticas (somente vegetação rasteira, formada por gramíneas e plantas herbáceas), e abióticas (maior variação microclimática em temperatura e umidade, e em luminosidade), fato que se refletiu 
significativamente no rol das famílias mais abundantes (ver adiante). A área fase 3, a mais afastada geograficamente, quase eqüidistante de todas as demais áreas, apresentou uma estrutura de fauna mais semelhante à da fase 1 , possivelmente devida a características físicas do folhiço, como a estrutura e densidade (quantidade das folhas, caules e galhos no solo), e os níveis de umidade, e não aos estágios de recomposição vegetal que apresentavam.

\section{Constância de famílias em diferentes áreas}

DAvies et al. (1997) observaram haver uma constância taxonômica na composição ao nível de família no dossel de floresta de várias regiões do globo. Por outro lado, CHUNG et al. (2000) comentaram haver uma diferença na composição de famílias dependendo da forma de coleta, com cada método amostrando determinadas famílias ou uma parte específica da comunidade de besouros.

A análise da estrutura taxonômica, nas áreas florestadas de Vila Velha, através da captura por armadilha de solo, foi semelhante àquela observada no folhiço de várias outras regiões do mundo, mesmo coletada por diferentes métodos, seja através de funil de Berlese, pelo aparato de Winkler, ou ainda pelo método de Kempson. Para evidenciar as relações entre as estruturas encontradas em um mesmo estrato florestal e as comparar àquelas que se reconhecem em outro estrato, pela captura através de armadilha malaise, foi feita uma análise de agrupamento, utilizando dados de presença de famílias de coleópteros de solo relacionadas em diferentes levantamentos realizados: em Ponta Grossa, no Paraná; no Amazonas (SCHUbarT \& BeCK 1968, Rodrigues 1992, Didham et al. 1998b, Barbosa et al. 2002); na Nova Zelândia (Moeed \& MeAds 1985); na Malásia (Chung et al. 2000), e os dados de presença de famílias em levantamentos feitos através de armadilha Malaise, no Paraná (MARINoni \& Dutra 1997) e na Nova Zelândia (Hutcheson 1990). Os resultados (figura 2; coeficiente de associação de Jaccard; UPGMA; coeficiente de correlação cofenética $=0,915$ ) corroboram as semelhanças da fauna de solo das várias regiões, diferindo da estrutura da fauna capturada por Malaise.

Estes padrões podem ser relacionados ao comportamento trófico das famílias que foram predominantemente herbívoras nos levantamentos feitos por Malaise no Paraná e apenas pouco inferiores às não-herbívoras na Nova Zelândia (HuTCHESON 1990); e predominantemente não-herbívoras (carnívoras, detritívoras, fungívoras) nos levantamentos da fauna de solo. Além do comportamento trófico, a diferença de captura nos dois estratos (na vegetação rasteira/sub-bosque e no solo), é uma decorrência dos hábitos relativos ao movimento pelo vôo (malaise), ou ambulatório (folhiço e armadilha de solo).

\section{Famílias mais abundantes}

Cerca de 90\% da abundância da fauna de Coleoptera de solo pode ser explicada pelas sete famílias mais abundantes (na área fase 3 este percentual chegou a 98\%). Nas áreas de borda interna e externa, os valores foram inferiores aos das demais áreas, com 82,3 e 93,3\%, respectivamente. A família mais abundante (Staphylinidae), apresentou percentuais entre 49 e 63\% (Tab. III) nas áreas florestadas, enquanto que a família dominante na borda externa foi Nitidulidae, não tendo atingido $30 \%$. Na maioria dos trabalhos o percentual das famílias mais abundantes situa-se acima de $85 \%$, como se verifica em

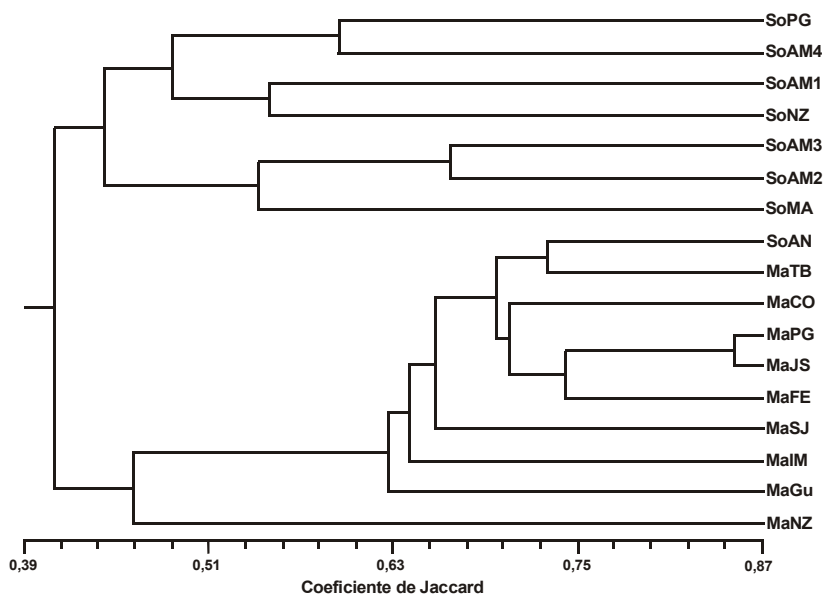

Figura 2. Análise de agrupamento. Relação entre diferentes localidades de diferentes regiões do mundo, com base na presença de famílias de Coleoptera, capturadas através de armadilha malaise (iniciais Ma) ou no solo (iniciais So). Dados extraídos de MARINONI \& Dutra (1997): MaAN (Antonina), MaCO (Colombo), MaFe (Fênix), MaGU (Guarapuava), MaJS (Jundiaí do Sul), MaPG (Ponta Grossa), MASJ (São José dos Pinhais), MaTB (Telêmaco Borba); Dutra \& MiYazakI (1995): MalM (Ilha do Mel); Hutcheson (1990), MaNZ (Nova Zelândia); com dados do presente estudo, SoPG (Ponta Grossa); de Schubart \& Beck (1968), SoAM1 (Amazonas); de Didham et al. (1998b), SoAM2 (Amazonas,); de Barbosa et al. (2002), SoAM3 (Amazonas); de Rodrigues (1992), SoAM4 (Amazonas); de Chung et al. (2000), SoMA (Malásia); de Moeed \& MeAds (1985), SoNZ (Nova Zelândia).

Schubart \& Beck 1968, Moeed \& Meads 1985, Rodrigues 1992, Carlton \& Robison (1998), Chung et al. 2000, Barbosa et al. 2002. Os dados constantes no trabalho de DidHAm et al (1998b), na Amazônia, no entanto, indicam que este valor está próximo de $66 \%$, diferindo de todos os demais levantamentos.

As famílias mais abundantes do solo, no conjunto das áreas de Vila Velha, foram Staphylinidade, Ptiliidae, Nitidulidae, Scarabaeidae, Scolytidae, Hydrophylidae e Endomychidae. O destaque em abundância nas áreas florestadas (araucária e fases 1,2 e 3) foram as famílias Staphylinidae e Ptiliidae.

$\mathrm{Na}$ área de borda externa, onde havia um predomínio de plantas herbáceas e gramíneas, e sem os elementos em decomposição do componente arbóreo (folhas, galhos, frutos) houve uma significativa alteração na composição das famílias mais abundantes (Tab. IV; coeficiente de correlação linear, $\mathrm{p}<0,05$ ). Nesta área, os Nitidulidae foram os mais abundantes, seguidos pelos Staphylinidae. Apareceram entre as famílias mais abundantes algumas que foram raras nas áreas florestadas, como Latridiidae, Corylophidae, Curculionidae. Os Ptiliidae, sempre a segunda família mais abundante nas áreas florestadas, foi pouco expressiva na borda externa, com apenas $2,5 \%$ da abundância total.

$\mathrm{Na}$ área de borda interna, dentro da mata, a $20 \mathrm{~m}$ da área de borda externa, ou seja, onde era menor a presença de herbáceas e gramíneas, e o folhiço constituía-se do material caído dos elementos arbóreos, a estrutura taxonômica foi semelhan- 
Tabela III. Famílias dominantes capturadas por armadilha de solo, ordenadas pelo percentual de abundância, em cinco áreas do Parque Estadual de Vila Velha, Ponta Grossa, Paraná, durante o período de setembro de 1999 a agosto de 2000.

\begin{tabular}{|c|c|c|c|c|c|c|c|c|c|c|c|}
\hline \multicolumn{2}{|c|}{ Borda externa } & \multicolumn{2}{|c|}{ Borda interna } & \multicolumn{2}{|c|}{ Araucária } & \multicolumn{2}{|l|}{ Fase 1} & \multicolumn{2}{|l|}{ Fase 2} & \multicolumn{2}{|l|}{ Fase 3} \\
\hline Família & (\%) & Família & (\%) & Família & (\%) & Família & (\%) & Família & (\%) & Família & (\%) \\
\hline Nitidulidae & 28,3 & Staphylinidae & 60,1 & Staphylinidae & 63,2 & Staphylinidae & 63,0 & Staphylinidae & 48,6 & Staphylinidae & 62,7 \\
\hline Staphylinidae & 25,1 & Ptiliidae & 9,7 & Ptiliidae & 17,6 & Ptiliidae & 23,4 & Ptiliidae & 21,0 & Ptiliidae & 22,0 \\
\hline Latridiidae & 11,8 & Nitidulidae & 9,6 & Nitidulidae & 5,8 & Scarabaeidae & 3,3 & Nitidulidae & 12,0 & Nitidulidae & 6,4 \\
\hline Scarabaeidae & 5,0 & Scolytidae & 5,6 & Scolytidae & 4,5 & Nitidulidae & 2,6 & Scolytidae & 5,9 & Scolytidae & 3,2 \\
\hline Corylophidae & 4,9 & Scarabaeidae & 4,3 & Scarabaeidae & 4,0 & Scolytidae & 1,5 & Scarabaeidae & 4,2 & Scarabaeidae & 2,2 \\
\hline Curculionidae & 4,0 & Hydrophilidae & 2,4 & Endomychidae & 1,7 & Endomychidae & 1,0 & Histeridae & 1,8 & Hydrophilidae & 1,0 \\
\hline Scolytidae & 3,2 & Carabidae & 1,6 & Histeridae & 1,0 & Hydrophilidae & 1,0 & Endomychidae & 1,3 & Endomychidae & 0,5 \\
\hline Total & 82,3 & & 93,3 & & 97,8 & & 95,8 & & 94,8 & & 98,0 \\
\hline
\end{tabular}

Tabela IV. Coeficiente de correlação linear entre dados das sete famílias mais abundantes de Coleoptera, coletadas por armadilha de solo, nos seis pontos de coleta, no Parque Estadual Vila Velha, Ponta Grossa, Paraná, durante o período de setembro de 1999 a agosto de 2000. (*) Correlação significativa $(p<0,05)$.

\begin{tabular}{|c|c|c|c|c|c|c|}
\hline \multirow{2}{*}{ Áreas } & \multicolumn{2}{|c|}{ Borda } & \multirow{2}{*}{ Araucária } & \multirow{2}{*}{ Fase 1} & \multirow{2}{*}{ Fase 2} & \multirow{2}{*}{ Fase 3} \\
\hline & Externa & linterna & & & & \\
\hline Borda Externa & - & & & & & \\
\hline Borda Interna & 0,6501 & - & & & & \\
\hline Araucária & 0,5655 & $0,9867^{*}$ & - & & & \\
\hline Fase 1 & 0,4996 & $0,9602^{*}$ & $0,9920^{*}$ & - & & \\
\hline Fase 2 & 0,6256 & $0,9592^{*}$ & $0,9812^{*}$ & $0,9807^{*}$ & - & \\
\hline Fase 3 & 0,5565 & 0,9725 * & $0,9958^{*}$ & 0,9970 * & $0,9906^{*}$ & - \\
\hline
\end{tabular}

te à das áreas florestadas, com os Staphylinidae sendo os mais abundantes. DiDHAm et al. (1998b) verificaram um aumento da abundância dos Staphylinidae (Pselaphinae) em direção à borda, creditando o fenômeno a um possível aumento de ácaros, que lhes serve de alimento; situação diversa foi observada em Vila Velha, onde a abundância foi muito menor na borda.

É razoável destacar que dentre as famílias mais abundantes, os Ptiliidae, juntamente com os Latridiidae e os Corylophidae, como grupos, foram mais sensíveis às modificações ambientais. Os Ptiliidae quase desapareceram na área de borda externa, enquanto que os Latridiidae e Corylophidae, pouco representados nas áreas florestadas, apareceram entre os mais abundantes, com percentuais de 12 e $5 \%$ respectivamente. As informações disponíveis na literatura indicam que tanto os Ptiliidae, como Latridiidae e Corylophidae, alimentam-se de fungos que se desenvolvem em folhiço. As diferenças de abundância podendo ser uma conseqüência das condições do folhiço, que difere muito da área de borda para as áreas florestadas, sejam pelas características orgânicas, seja pela umidade. $\mathrm{O}$ fato de Ptiliidae ter tido uma abundância muito superior na área florestada pode ser explicada pela maior umi- dade do folhiço e menor luminosidade ocorrente no solo das áreas florestadas, com conseqüente proliferação de fungos. Na Amazônia, onde se caracteriza nitidamente a existência de duas estações, uma chuvosa e uma seca, houve um grande aumento da abundância desta família na época chuvosa (RodRIGUes 1992); a disparidade de abundância de Ptiliidae foi destacada por BARBOSA et al. (2002).

Dentre as famílias reconhecidas como as sete mais abundantes no solo, conforme estudos citados acima, destacam-se Staphylinidae, Scolytidae, Scydmaenidae, Carabidae, Ptiliidae, Curculionidae, Nitidulidae, Scarabaeidae e Leiodidae.

As famílias com maior percentual de abundância foram as não-herbívoras. Staphylinidae, a mais abundante, atingindo picos de cerca de $65 \%$ do total de indivíduos capturados em alguns dos levantamentos, inclui predominantemente espécies carnívoras e, em menor número, fungívoras e detritívoras. Scydmaenidae e Carabidae, famílias fundamentalmente carnívoras, situaram-se entre as mais abundantes na maioria dos levantamentos. Scydmaenidae foi abundante nos levantamentos nas áreas tropicais (Amazonas e Malásia), mas pouco expressiva nas áreas temperadas, como observado em Ponta Grossa e na Nova Zelândia (Orongorongo), exceto em Ouachita, no oeste do Arkansas (Estados Unidos da América), onde atingiu a expressiva abundância de 8\% (CARLTON \& RoBISON 1998). A menor presença de Scydmaenidae nas áreas temperadas do Hemisfério Sul pode estar ligada a características zoogeográficas. Nas áreas tropicais florestadas, há substituição de parte da abundância de Staphylinidae pelos Scydmaenidae. Em Vila Velha, a abundância de Staphylinidae é de cerca de $60 \%$, com rara presença de Scydmaenidae ( $<1 \%$ ), enquanto que nas áreas tropicais são responsáveis por apenas 35 a 50\% da abundância, com os Scydmaenidae atingindo até cerca de $22 \%$, no Amazonas (Rodrigues 1992). Neste processo de oscilação da abundância dos carnívoros, os Carabidae contribuem com um percentual que atinge até $7 \%$. Scolytidae, família com hábitos alimentares variados (xilófagos, xilomicetófagos, mielófagos, fleófagos, mielófagos, espermófagos e fungívoros), foi anotada entre as sete mais abundantes em quase todos os levantamentos; mas, o maior percentual de abundância não atinge a 13\%, com a maioria dos levantamentos indicando uma abundância em torno de $4 \%$. Os Ptiliidae, fungívoros, foram abundantes nas 
áreas florestadas de Vila Velha, em dois levantamentos no Amazonas (Schubart \& Beck 1968, Rodrigues 1992), e em Ouachita, no Arkansas (CARLTON \& RoBison 1998). Nas localidades onde diminuiu a abundância desta família, observou-se um aumento na abundância de outras, como Leiodidae (DiDHAM et al. 1998b, Moeed \& Meads 1985, Chung et al. 2000); de outras apenas eventualmente presentes, como Melandryidae e Tenebrionidae (BARBosa et al. 2002) e Latridiidae, Corylophidae, no levantamento em Vila Velha. Estas famílias tendo também a maioria das espécies com hábitos fungívoros. Este fato podendo indicar um processo de substituição de táxons do mesmo grupo trófico adaptados a diferentes condições orgânicas e físicas do folhiço, quando numa mesma área, ou zoogeográfico, quando em diferentes regiões.

Dentre as famílias unicamente herbívoras, apenas os Curculionidae aparecem eventualmente entre os mais abundantes, exceto no estudo em Orongorongo (Nova Zelândia) (Moeed \& Meads 1985). Nesse estudo (Moeed \& Meads 1985), em quatro diferentes pontos de coleta, a família foi a mais abundante em três deles, com percentual muito alto (até 38\%); e não há elementos para explicar uma possível causa de tal abundância, quando comparada às abundâncias de todos os demais levantamentos referidos neste trabalho, sempre inferiores a $7 \%$.

Como discutido anteriormente, a composição taxonômica ao nível de família é mais semelhante quando comparada às de regiões diferentes, que àquelas dos diferentes estratos de uma floresta de uma mesma área coletadas por métodos diferentes. Este fato também foi observado nos estudos em Vila Velha.

Porém, como indicam os dados da tabela $\mathrm{V}$, as diferenças entre os valores de abundância das sete famílias mais abundantes têm maior expressão que a diferença na composição de famílias, em cada um dos métodos de coleta. Na armadilha malaise foram mais abundantes as famílias Chrysomelidae, Mordelidae, Cerambycidae, Elateridae, Curculionidae e Staphylinidae, ou seja, 6 famílias com cerca de $60 \%$ do total de indivíduos; e na armadilha de solo foram mais abundantes as famílias Staphylinidae, Ptiliidae, Nitidulidae, Scarabaeidae e Scolytidae, com as duas primeiras sendo responsáveis por cerca de $75 \%$ do total. Todas estas famílias com abundâncias fortemente diferenciadas em cada um dos ambientes. Em termos tróficos, aumenta a abundância de famílias herbívoras nas coletas por malaise, e de carnívoras, fungívoras, detritívoras no solo. Além do hábito trófico para caracterizar a fauna de Coleoptera dos dois extratos do ambiente, sub-bosque e solo, devem ser destacadas as suas características locomotoras, que é voadora na captura por malaise, e ambulatória na captura por armadilha de solo.

\section{CONSIDERAÇÕES FINAIS}

As análises dos dados de abundância e riqueza de famílias de Coleoptera que habitam o solo indicam que:

Em Vila Velha, Ponta Grossa: 1) a abundância dos coleópteros do solo nas áreas florestadas é muito superior à da área de borda; e na área florestada em estágio de sucessão inicial é superior às das áreas em estágio intermediário e avançado; 2) não há diferença significativa na composição da fauna de Coleoptera do solo, nas diferentes áreas; porém quando são arroladas apenas as sete mais abundantes, observa-se uma composição significativamente diferente da área de borda com re-
Tabela V. Percentual de abundância das famílias dominantes de Coleoptera, através de dois métodos de coleta, no Parque Estadual de Vila Velha, Ponta Grossa, Paraná, durante o período de setembro de 1999 a agosto de 2000.

\begin{tabular}{lcc}
\hline \multicolumn{1}{c}{ Famílias dominantes } & Solo (\%) & Malaise (\%) \\
\hline Staphylinidae & 58 & 7 \\
Ptiliidae & 19 & $<1$ \\
Chrysomelidae & $<1$ & 16 \\
Nitidulidae & 8 & 3 \\
Mordelidae & $<1$ & 12 \\
Scarabaeidae & 4 & 6 \\
Cerambycidae & $<1$ & 9 \\
Elateridae & $<1$ & 8 \\
Curculionidae & 1 & 7 \\
Scolytidae & 4 & 2 \\
Ptilodactylidae & $<1$ & 4 \\
Cleridae & $<1$ & 4 \\
Endomychidae & 1 & 1 \\
Hydrophilidae & 1 & $<1$ \\
Latridiidae & 1 & $<1$ \\
Subtotal para 15 famílias acima & 97 & 79 \\
Demais 49 famílias em malaise & & 21 \\
Demais 20 famílias em solo & 3 & \\
\hline
\end{tabular}

lação às demais; 3) as características da fauna capturada por malaise estão mais relacionadas às características vegetais das áreas, enquanto que a fauna de solo está mais relacionada às características do folhiço, que além de ter sua constituição dependente da vegetação é influenciado pela umidade.

Em Vila Velha, Ponta Grossa, como também em diferentes regiões do mundo: 1) a riqueza de famílias capturadas no solo é inferior à riqueza através da captura por armadilha malaise; 2) a composição taxonômica ao nível de família no solo é mais assemelhada entre si, mesmo em diferentes regiões zoogeográficas, que à composição de famílias capturadas por malaise nas mesmas áreas; 3 ) cinco a sete famílias mais abundantes respondem por cerca de $90 \%$ da abundância total da fauna de coleópteros de solo, como observado na maioria dos levantamentos; e por $60 \%$ da abundância quando capturada por armadilha malaise; 4) entre as famílias mais abundantes no solo de Vila Velha, estão Staphylinidae, Ptiliidae, Nitidulidae, Scarabaeidae, Scolytidae, Hydrophilidae e Endomychidae, eventualmente substituídas por Latridiidae, Corylophidae, Curculionidae, Carabidae e Histeridae; enquanto que nos demais levantamentos houve o registro de Staphylinidae, Ptiliidae, Nitidulidae, Scolytidae, Scydmaenidae, Carabidae, Curculionidae, Scarabaeidae e Leiodidae. 5) na fauna de solo há uma dominância de famílias carnívoras, fungívoras e detritívoras, e de famílias herbívoras na fauna capturada por malaise; esta relação ocorreu de forma inversa nos estudos na 
Nova Zelândia, onde Curculionidae, com hábitos herbívoros, foi a família mais abundante no solo (Moeed \& Meads 1985), e as famílias não-herbívoras dominaram nas capturas por malaise (Hutcheson 1990); 6) táxon de um mesmo grupo trófico é substituído por outro do mesmo grupo - a) em uma mesma região, possivelmente por estar mais adaptado às condições orgânicas e físicas do folhiço, como entre os fungívoros Ptiliidae, Latridiidae, Corylophidae, e - b) em regiões diferentes, pela condição zoogeográfica, como entre os carnívoros Staphylinidae, Scydmaenidae e Carabidae, e entre os fungívoros Leiodidae e Melandryidae.

\section{AGRADECIMENTOS}

Renovamos os nossos agradecimentos já externados em GANHO \& MARINONI (2003) a Rosina Djunko Miyazaki (Universidade Federal do Mato Grosso), Germano Rosado Neto, José Ricardo Mermudes, Keila Trefflich, Franklin Galvão, Yoshiko Saito Kuniyoshi, Armando Cervi, Edílson Caron, Adelita Linzmeier (Universidade Federal do Paraná) e Josemar Marins (Instituto Agronômico do Paraná), pelas várias formas de colaboração prestadas. Ao Instituto Agronômico do Paraná (IAPAR) e ao Instituto Brasileiro do Meio Ambiente e dos Recursos Naturais Renováveis (IBAMA) pela autorização de coleta no Parque Estadual de Vila Velha; à Coordenação de Aperfeiçoamento de Pessoal e Ensino Superior (CAPES); ao Conselho Nacional de Desenvolvimento Científico e Tecnológico (CNPq) e à Fundação da Universidade Federal do Paraná (FUNPAR), pela concessão de bolsas e auxílio financeiro.

\section{REFERÊNCIAS BIBLIOGRÁFICAS}

Almeida, L.M.; C.S.Ribeiro-Costa \& L. Marinoni. 1998. Manual de coleta, conservação, montagem e identificação de insetos. Ribeirão Preto, Holos, 78p.

Barbosa, M.G.V.; C.R.V. Fonseca; P.M. Hammond \& N.E. Stork. 2002. Diversidade e similaridade entre habitats com base na fauna de Coleoptera de serrapilheira de uma floresta de terra firme da Amazônia Central, p. 69-83. In: C. Costa; S.A. Vanin; J.M. Lobo \& A. Melic (Eds). Proyecto de Red Iberoamericana de Biogeografía y Entomología Sistemática. Inventarios y Biodiversidad de insectos. Zaragoza, Gorfi, vol. 2, 329p.

Carlton, C.E. \& H.W. Robison. 1998. Diversity of litter-dwelling beetles in the Ouachita highlands of Arkansas, USA (Insecta: Coleoptera). Biodiversity and Conservation, Londres, 7: 1589-1605.

Costa, C. 2000. Estado de conocimento de los Coleoptera neotropicales, p. 99-114. In: F.M. Piera,; J.J. Morrone \& A. MELIC (Eds). Hacia um proyecto CYTED para el inventario y estimación de la diversidad entomológica em Iberamérica: PrIBES2000. Zaragoza, Gorfi, 326p.

Chung, A.Y.C.; P. Eggleton, M.R. Speight; P.M. Hammond \& V.K. CHey. 2000. The diversity of beetle assemblages in different habitat types in Sabah, Malaysia. Bulletin of Entomological Research, Farnham Royal, 90: 475-496.
Davies, J.G.; N.E. Stork; M.J.D. Brendell \& S.J. Hine. 1997. Beetle species diversity and faunal similarity in Venezuelan rainforest tree canopies, p. 85-103. In: S.N.E. TORK; J. ADIS \& R.K. Didham (Eds). Canopy Arthropods. Londres, Chapman $\&$ Hall, 567p.

Didham, R.K.; P.M. HAMmond; J.H. LAWTON; P. EgGLeton \& N.E.STORK. 1998a. Beetle species responses to tropical forest fragmentation. Ecological Monographs, Lawrence, 68 (3): 295-323.

Didham, R.K.; J.H. Lawton; P.M. Hammond \& P. EgGleton. 1998b. Trophic structure stability and extinction dynamics of beetles (Coleoptera) in tropical forest fragments. Philosophical Transactions of Royal Society of London 353: 437-451.

Dutra, R.R.C. \& R.D. MiYazaKI. 1995. Famílias de Coleoptera capturadas com armadilha malaise em duas localidades da Ilha do Mel, Baía de Paranaguá, Paraná, Brasil. Arquivos de Biologia e Tecnologia, Curitiba, 38 (1): 175-190.

Ganho, N.G. \& R.C.Marinoni. Fauna de Coleoptera no Parque Estadual de Vila Velha, Ponta Grossa, Paraná, Brasil. Abundância e riqueza das famílias capturadas através de armadilhas malaise. Revista Brasileira de Zoologia, Curitiba, 20 (4): 727-736.

Hutcheson, J. 1990. Characterization of terrestrial insect communities using quantified, Malaise-trapped Coleoptera. Ecological Entomology, Londres, 15: 143-151.

Lawrence, J.F. \& A.F. Newton. 1995. Families and subfamilies de Coleoptera (With select genera, notes, references and data on family-group names), p. 779-1006. In: J.F. PAPALUK \& S.A. SLIPINSKI (Eds). Biology, phylogeny, and classification of Coleoptera. Varsóvia, Museum i Institut Zoologii PAN, 1092p.

Marinoni, R.C. \& R.R.C. Dutra. 1997. Famílias de Coleoptera capturadas com armadilha malaise em oito localidades do Estado do Paraná, Brasil. Diversidades alfa e beta. Revista Brasileira de Zoologia, Curitiba, 14 (3): 751-770.

Marinoni, R.C.; N.G. Ganho; M.L. Monné \& J.R.M. Mermudes. 2001. Hábitos alimentares em Coleoptera (Insecta). Ribeirão Preto, Holos, 63p.

Moeed, A. \& M.J. Meads. 1985. Seasonality of pitfall trapped invertebrates in three types of native forest, Orongorongo Valley, New Zealand. New Zealand Journal of Zoology, Wellington, 12: 17-53.

Ozanne, C.M.P.; C. Hambler; A. Foggo \& M.R. Speight. 1997. The significance of edge effects in the management of forests for invertebrate biodiversity, p. 534-550. In: N.E. STORK; J. ADIs \& R.K. Didham (Eds). Canopy Arthropods. Londres, Chapman \& Hall, 567p.

Rodrigues, J.M.G. 1992. Abundância e distribuição vertical de coleópteros do solo em capoeira de Terra Firme, na região de Manaus - AM, Brasil. Acta Amazonica, Manaus, 22 (3): 323-333.

Rohlf, F.J. 1998. NTSYSpc. Numerical Taxonomy and Multivariate Analysis System. Version 2.02i. New York, Exeter Publ.Ltd.

Schubart, H. \& L.Beck. 1968. Zur Coleopterenfauna amazonischer Böden. Amazoniana, Kiel, 1 (4): 311-322.

Recebido em 24.VI.2003; aceito em 17.XI.2003.

Revista Brasileira de Zoologia 20 (4): 737-744, dezembro 2003 